\section{PWE-046 SYSTEMATIC REVIEW: CLOSTRIDIUM DIFFICILE AND IBD}

doi:10.1136/gut.2011.239301.309

J R Goodhand, "W Alazawi, D Rampton Gastroenterology, Barts and the London NHS Trust, London, UK

Introduction There is increasing concern about the apparently rising incidence and worsening outcome of clostridium difficile infection (CDI) associated with inflammatory bowel disease (IBD). We have systematically reviewed, using PRISMA guidelines, the literature to evaluate the incidence risk factors, endoscopic features, treatment and outcome of CDI complicating IBD.

Methods Structured searches of PubMed up to September 2010 for cross-sectional, cohort and case controlled studies were undertaken.

Results Of 407 studies identified, 42 met the inclusion criteria: their heterogeneity precluded formal meta-analysis. There were no significant differences between the weighted mean incidence (weighted $95 \% \mathrm{CI}$ ) in adult patients with UC or Crohn's disease (CD) by either culture (UC 6.7\% (5.6-11.4), Crohn's disease 8.8\% (2.5-15.1)) or toxin assay (UC 3.1\% (0.16.2), Crohn's disease 7.0\% (1.8-12.2)). The weighted mean incidence of CDI, derived from two studies of children in relapse was significantly higher than in adults $26.1 \%$ (16.1-36.1). In remission, patients with IBD are more frequently colonised than otherwise healthy controls, the number of strains implicated suggests multiple community sources of acquisition. Certainty about a temporal trend to an increasing incidence of CDI in IBD, reportedly complicating about $3.9 \%$ and $1.2 \%$ admissions for UC and Crohn's disease in 2004, increasing to $5.3 \%$ and $1.5 \%$ respectively in 2008 , is compromised by detection bias as a result of more frequent testing and miscoding. Antibiotics are less of a pre-requisite for CDI in IBD than in the general population: where reported only $43 \%$ (23/54) toxinpositive relapse samples were associated with antibiotic usage. Similarly nosocomial acquisition is reportedly less frequently than in the general population: where reported nosocomial acquisition was suspected in only $26 \%(5 / 19)$ patients with toxin-positive relapses. Further risk factors for acquisition, like in control populations include increasing age, immunosuppressant and colonic disease involvement. 7 studies reported on the use of endoscopy in IBD with CDI: only 9\% (13/150) patients had pseudomembranes. There are no controlled trials of CDI treatment IBD. Contradictory data, probably accounted for by differences in trial design, are reported for length of stay, colectomy and mortalities.

Conclusion Patients with IBD seem to be at increased risk for CDI compared to controls and CDI in IBD has received increasing attention in the past decade, but whether its incidence is really increasing or its outcome worsening remains unproven. Endoscopy is unhelpful in diagnosing CDI in IBD. Therapeutic trials of CDI treatment are lacking and are urgently needed.

Competing interests None. 